\title{
Concepciones sobre la naturaleza de la ciencia en el profesorado en formación inicial
}

\section{Pre-service teachers' conceptions of the nature of science}

José Francisco Serrallé Marzoa, Uxío Pérez Rodríguez, María Asunción Lorenzo Rial, María Mercedes Álvarez Lires Facultad de Ciencias de la Educación y del Deporte. Universidade de Vigo. A Xunqueira, s/n, 36005-Pontevedra jfserralle@uvigo.es, uxio.perez@uvigo.es, marialorenzo@uvigo.es, lires@uvigo.es

RESUMEN • Se ha diseñado y validado un cuestionario, a fin de indagar sobre las concepciones acerca de la ciencia y su naturaleza (NOS) en el profesorado en formación inicial, a partir de cuyos resultados se diseñarán posteriores intervenciones didácticas sostenidas de enseñanza de la NOS en el aula. Se describe el cuestionario, la muestra $(\mathrm{N}=385)$ y el procedimiento de validación. La escala tiene una fiabilidad y validez correctas, por lo que se concluye que puede ser de utilidad para la investigación relacionada con esta temática. También se realiza un análisis clúster de las puntuaciones en los factores de la escala, con el fin de segmentar los casos en grupos de perfiles de respuestas similares y elaborar «Tipos docentes».

PALABRAS CLAVE: Formación inicial del profesorado; Concepciones; NOS; Validación cuestionario; Tipos docentes.

ABSTRACT • A questionnaire has been designed and validated to investigate the conceptions about science and its nature (NOS) among teachers in initial training, from whose results subsequent didactic interventions of teaching NOS in the classroom will be designed. The questionnaire, the sample $(\mathrm{N}=385)$ and the validation procedure are described. The scale has a correct reliability and validity, so it is concluded that it can be useful for research related to this topic. A cluster analysis of the scale factor scores is also carried out, so as to segment the cases into groups of similar response profiles and to draw up «Teaching Types».

KEYWORDS: Teacher training; Conceptions; NOS; Questionnaire validation; Teaching types. 


\section{INTRODUCCIÓN}

Desde hace más de cuatro décadas, numerosos trabajos de investigación han puesto de manifiesto, con carácter general, que la enseñanza en las etapas de educación primaria y secundaria se realiza con una perspectiva y un tratamiento muy formales, excesivamente declarativo de los contenidos científicos, que presentan el conocimiento científico como una verdad inmutable, como un resultado cerrado de sucesivos descubrimientos acumulativos y a modo de constante perfeccionamiento lineal de modelos y de teorías; incluso se afirma la existencia de un único método de hacer ciencia: «el método científico» (Serrallé, 2018).

Por otra parte, diversas investigaciones indican que el profesorado posee concepciones, modelos, imágenes y creencias que influyen en su manera de promover el aprendizaje y de enseñar que son transferidas a su alumnado (Ravanal, Joglar, Quintanilla y Labarrere, 2009). Estas concepciones sobre la ciencia, la forma de aprenderla y enseñarla, y sobre los contenidos que se deben enseñar se basan en sus experiencias anteriores y actuales e incluyen características explícitas del currículo, conocimientos de carácter conceptual, procedimental y actitudinal, pero también en aspectos menos explícitos como sus valores, la forma de evaluar, la dinámica social del aula, las emociones y los sentimientos que les generaron esas experiencias (Bonil y Márquez, 2011). Dichas concepciones son persistentes, de difícil modificación (Arias Correa, 2012), y actúan como instrumentos que sirven para interpretar la realidad, en este caso, de la enseñanza de las ciencias, de manera que configuran modelos didácticos (Angulo, 2002). Pero también constituyen barreras u obstáculos que dificultan la adopción de nuevos puntos de vista (Bachelard, 1938; Claxton, 1994; Pope y Gilbert, 1983; Porlán, Díaz y de León, 1988). En este sentido, Zimermann (2000) apunta que los modelos didácticos del profesorado condicionan el tipo de tareas que propone en el aula.

Las concepciones del profesorado sobre la naturaleza de la ciencia y la tecnología (NOS) y sus visiones acerca de las relaciones entre ellas y con la sociedad, así como de la producción del conocimiento científico, interaccionan con su forma de enseñar y con sus ideas sobre el aprendizaje del alumnado (Ferreira-Gauchía, Vilches y Gil-Pérez, 2012). Cuando el futuro profesorado, durante su formación universitaria, no logra reformular sus creencias o cogniciones previas sobre la ciencia, para elaborar un modelo docente propio, tiende a repetir la metodología de enseñanza recibida previamente (Brandáo, 2008). Se bloquea, así, la evolución hacia nuevos paradigmas didácticos, la innovación pedagógica e incluso la necesaria autoformación docente en el posterior ejercicio profesional (Martín, Prieto y Jiménez, 2015).

El futuro profesorado será el responsable de formular situaciones de aprendizaje en el aula que propicien una «descripción precisa y detallada de las funciones, procesos, métodos y límites de la ciencia»; argumentar el conocimiento de la naturaleza de la ciencia posibilitará al alumnado aprendizajes significativos. En tal sentido, es preciso investigar y determinar las creencias sobre la ciencia del alumnado -profesorado en formación-, ya que van a condicionar la intervención docente y pueden limitarla (Arias Correa, 2012; McComas, Clough y Almazroa, 1998).

Pese a lo expresado hasta ahora, las concepciones alternativas no han de ser vistas, únicamente, como un obstáculo epistemológico (Bachelard, 1938) o un impedimento al aprendizaje, sino como un punto de partida que se ha de conocer para construir los nuevos conocimientos científicos (Duit, Gropengieber y Kattmann, 2005).

Antes de continuar, se ha de señalar, siguiendo a Furió, Solbes y Carrascosa (2004), que en la década de los ochenta se utilizaba toda una gama de términos para nombrar las concepciones alternativas, como ideas alternativas, preconceptos, preconcepciones, ideas previas, ideas alternativas, teorías implícitas, etc. Wandersee, Mintzes y Novak (1994) propusieron la denominación genérica de concepciones 
alternativas, que hoy es la más aceptada. En lo que sigue, se utilizará la denominación concepciones sobre la naturaleza de la ciencia (NOS conceptions).

El término NOS se acuñó en 1907 en la Central Association of Science and Mathematics Teachers. Desde entonces, la comprensión de la NOS ha sido un objetivo educativo, «cuya longevidad ha sido superada sólo por la longevidad de la incapacidad del estudiantado para articular el significado de la naturaleza de la ciencia, y para identificar las características asociadas a la ciencia» (Lederman y Niess, 1997).

Según McComas, Clough y Almazroa (1998) y Clough (2006), el acrónimo NOS se utiliza para referirse a cuestiones tales como: $a$ ) ¿qué es y qué no es ciencia?, b) ¿cómo se trabaja en la ciencia?, $c$ ) ¿cuáles son los fundamentos ontológicos y epistemológicos de la ciencia? y d) ¿cómo interaccionan ciencia, tecnología y sociedad?

Por su parte, Adúriz-Bravo (2005) indica que la NOS se puede considerar como un conjunto de contenidos metacientíficos seleccionados por su valor para la educación científica de la ciudadanía, sobre los cuales se realiza una adecuada transposición didáctica (Adúriz-Bravo, 2005). Y, así, Izquierdo, García, Quintanilla y Adúriz (2016) entienden la NOS como un conjunto de contenidos procedentes de diversas escuelas de la filosofía de la ciencia, «ambientados en episodios paradigmáticos de la historia de la ciencia y advertidos por la sociología de la ciencia contra el dogmatismo, el triunfalismo, el elitismo y el sesgo de género propios de las visiones de ciencia tradicionales, comúnmente calificadas de cientificistas o tecnocráticas» (Adúriz-Bravo, 2006, 2008).

Driver, Leach, Millar y Scott (1996) señalan cinco aspectos acerca de la importancia de comprender la NOS: $a$ ) dar sentido a la ciencia y manejar objetos y procesos tecnológicos en la vida cotidiana (utilitaria); $b$ ) tomar decisiones informadas en cuestiones sociocientíficas (democrática); $c$ ) apreciar el valor de la ciencia como parte de la cultura contemporánea (cultural); d) ayudar a comprender las normas de la comunidad científica, de acuerdo con compromisos morales válidos para la sociedad (moral); f) facilitar el aprendizaje de las ciencias.

No existe un acuerdo unívoco sobra la conceptualización de la NOS, si bien Lederman, AbdEl-Khalick, Bell y Schwartz (2002) describen las «creencias informadas» acerca de la ciencia como conocimientos científicos que el colectivo de estudiantes debe adquirir. Lederman (2007) afirma que existe bastante acuerdo entre la filosofía de la ciencia, la historia de la ciencia y la didáctica de las ciencias sobre un conjunto de cuestiones referentes a la naturaleza de la ciencia: $a$ ) el conocimiento de la ciencia es empírico; $b$ ) las observaciones y las inferencias difieren; $c$ ) las teorías científicas son sistemas explicativos internamente consistentes que guían las investigaciones y el descubrimiento; $d$ ) las leyes representan relaciones entre variables cuantificables; $e$ ) la ciencia se basa en la imaginación y en la creatividad humana y no siempre es racional; $f$ la ciencia está cargada de teoría y, en consecuencia, las observaciones no son objetivas; $g$ ) la ciencia es una empresa humana incrustada en una cultura social; h) no hay un método científico único; e $i$ ) el conocimiento científico es provisional y holístico.

\section{Las creencias del profesorado sobre la NOS}

El conocimiento de la NOS es condición necesaria pero no suficiente para que dicho conocimiento se refleje en la práctica en el aula, ya que este proceso está mediado por un complejo conjunto de variables que aún no se ha resuelto totalmente y que tienen que ver, entre otros aspectos, con las metodologías del aula o la utilización de la modelización (Justi y Gilbert, 2002). Es necesario, además, identificar los factores que influyen en las concepciones del colectivo estudiantil. Sin embargo, es preciso decir que hay acuerdo en las investigaciones acerca de la fuerte influencia de las limitaciones de los planes de estudios, políticas administrativas y el contexto de la enseñanza sobre la traducción de las concepciones sobre la NOS en la práctica del aula (Lederman, 2007, 2018). 


\section{Las creencias del profesorado sobre la NOS}

Schwartz y Lederman (2008) encontraron variaciones en las creencias sobre la NOS, que clasifican en «informadas, parcialmente informadas e ingenuas». Las creencias informadas coinciden con muchos de los aspectos que se han indicado al hablar del acuerdo existente en la comunidad científica sobre la NOS.

Lederman, Abd-El-Khalick, Bell y Schwartz (2002) enumeran las creencias ingenuas sobre la ciencia: a) los hechos establecidos, a través de la evidencia empírica, generan la base de conocimiento; $b$ ) constituye la búsqueda de la verdad objetiva sobre el mundo; $c$ ) se basa en la observación directa; $d$ ) utiliza un único método científico (tácitamente acordado) cuasi algorítmico; $e$ ) no requiere la creatividad ni la imaginación; $f$ impulsa el cambio en las teorías por acumulación reduccionista de pruebas; g) permite que las teorías se conviertan en leyes por la repetición de las pruebas; $h$ ) es independiente de factores sociales y culturales.

Abd-El-Khalick y Akerson (2009) describen las creencias parcialmente informadas en las que se combinan aspectos de las creencias informadas y de las creencias ingenuas. Por ejemplo, reconocen la ciencia como sujeto empírico que implica observaciones, haciendo y probando predicciones, pero invocan un mecanismo simplista para la toma de datos y no reconocen que los conocimientos adquiridos son provisionales; admiten una escasa influencia de la imaginación y de la creatividad.

Según el grupo de pertenencia, la atención que se le presta a la enseńanza de la NOS es decreciente, desde el grupo que posee creencias informadas hasta el poseedor de creencias ingenuas. Parece, pues, evidente que las creencias sobre la NOS tienen una influencia directa en la práctica docente del profesorado, junto con sus modelos de docencia, aprendizaje del alumnado y los currículos.

Desde los años cincuenta se han desarrollado investigaciones sobre concepciones relacionadas con la NOS en profesorado y alumnado (Lederman, 2007). Se ha encontrado que, a pesar del tiempo transcurrido, las creencias ingenuas han permanecido casi inmutables y ello pese a que la ciencia actual ya no es, obviamente, la de los siglos XvII o XvIII, pero ni siquiera la del siglo xx, y hoy habría que referirse a la tecnociencia (Echeverría y González, 2009). En investigaciones cualitativas se ha encontrado que el profesorado en formación inicial, en su mayoría, no percibe las relaciones entre ciencia, tecnología y sociedad, ni tampoco comparte la concepción de ciencia como actividad humana, inherente a la tecnociencia (Álvarez-Lires, Arias, Pérez-Rodríguez y Serrallé, 2013).

Para indagar en las concepciones del profesorado en formación inicial del grado en Educación Primaria sobre la NOS se llevó a cabo el diseño y la validación de un cuestionario sobre la naturaleza de la ciencia (NOS), con la finalidad de evaluarlas y, posteriormente, partir de ellas para llevar a cabo propuestas didácticas de aula.

\section{METODOLOGÍA}

Inicialmente, se llevó a cabo una revisión de la literatura especializada en cuestionarios sobre la NOS (Serrallé, 2018) y bibliografía sobre concepciones de estudiantes y profesorado (Duit, 2009). Se encontraron dos tendencias: a) cuestionarios con preguntas abiertas y, en ocasiones, complementados con entrevistas, y b) cuestionarios con preguntas o ítems cerrados. Lederman (2007) realizó una revisión de 30 cuestionarios validados de este tipo de investigaciones, desde el año 1954 al 2006, y Duit (2009) realizó una recopilación bibliográfica desde 1952 hasta 2008, ambas referidas al ámbito anglosajón. Para la presente investigación se han utilizado, además, las de Porlán, Rivero y Martín (1997), Briceño y Benarroch (2009) y Brandão, Araujo, Veit y Lang (2011). El análisis detallado de los resultados de cada una de las investigaciones excede las posibilidades de extensión de este artículo. 


\section{Diseño del cuestionario inicial}

Para la elaboración del cuestionario anónimo empleado en la investigación se realizaron reuniones de personas expertas que propusieron ítems. En una primera selección se formuló un cuestionario piloto tipo Likert compuesto por 50 ítems de tipo ordinal con cuatro niveles de respuesta, el cual fue administrado a una pequeña muestra de alumnado del grado en Educación Primaria. Teniendo en cuenta los resultados del estudio exploratorio se reformuló el cuestionario, se eliminaron cuestiones problemáticas por motivos estadísticos (ítems que hacían descender la fiabilidad del instrumento, problemas de multicolinealidad, ítems que no saturaban claramente en ningún factor) y se reescribieron algunas preguntas que el alumnado de la prueba piloto tenía problemas para responder. La versión definitiva fue una escala (Blanco, Sanz y Vallejo, 2003) de 44 ítems en los que se debe manifestar el grado de acuerdo o desacuerdo, optando selectivamente entre uno de los cuatro niveles propuestos, mediante un número par de ítems para evitar la tendencia a la centralidad (Cummins y Gullone, 2000). Las categorías tratadas en dichos ítems se han elaborado basándose en la revisión efectuada por Serrallé (2018) y se muestran en la tabla 1.

Tabla 1.

Temáticas abarcadas por los ítems del cuestionario inicial

\begin{tabular}{|l|}
\hline Positivismo científico \\
\hline Hermenéutica científica \\
\hline Metaciencia \\
\hline Igualdad de género en la ciencia \\
\hline Personas dedicadas a la ciencia \\
\hline Metodologías científicas \\
\hline Aplicabilidad de la ciencia \\
\hline Visión de la ciencia \\
\hline Interacción ciencia - tecnología - sociedad - medioambiente \\
\hline
\end{tabular}

\section{Muestra y procedimiento de administración}

La muestra utilizada para la validación del cuestionario CIPT estuvo compuesta por 385 estudiantes del grado en Educación Primaria de la Facultad de Ciencias de la Educación y del Deporte de Pontevedra, 313 mujeres y 72 hombres (81 y $19 \%$ de la muestra, respectivamente). El cuestionario se administró durante cinco años consecutivos al alumnado de la asignatura «Ciencias experimentales», impartida en segundo curso de la titulación, al comienzo de la docencia. La repetición en años consecutivos busca asegurar la sistematización en la recopilación de los datos. La elección de pasar el cuestionario al iniciar la primera sesión lectiva trata de evitar las posibles repercusiones que cualquier acción docente pudiera tener, y que pudiera influir en las respuestas.

El cuestionario anónimo se rellena, en los dos primeros ańos, en el aula, impreso en papel, para observar posibles incidencias o dificultades, y en los tres años siguientes, telemáticamente, on line, utilizando la aplicación «Formularios de Google». 


\section{Validación y calibración del cuestionario}

La muestra original $(\mathrm{N}=385)$ se dividió en dos submuestras aleatorias (muestra de validación $\mathrm{N}_{\mathrm{V}}=$ 192 y de calibración $\mathrm{N}_{\mathrm{C}}=193$ ). Con la primera se realizó un análisis exploratorio para elaborar un modelo de las principales dimensiones implicadas y la segunda se utilizó para llevar a cabo un análisis factorial confirmatorio.

Para validar el instrumento se realizó un análisis descriptivo, de consistencia interna y exploratorio utilizando el software SPSS 20 para Windows. El análisis factorial confirmatorio se llevó a cabo con el programa AMOS 21, y se empleó Factor 9.3 para el cálculo de los coeficientes glb y $\Omega$ y para el estudio del número de factores que deben extraerse.

Tras ello se llevó a cabo la elaboración del cuestionario definitivo, del que se presentan evidencias de fiabilidad y validez, y se analiza la estructura factorial subyacente.

Los pasos seguidos en el estudio se resumen en la figura 1.

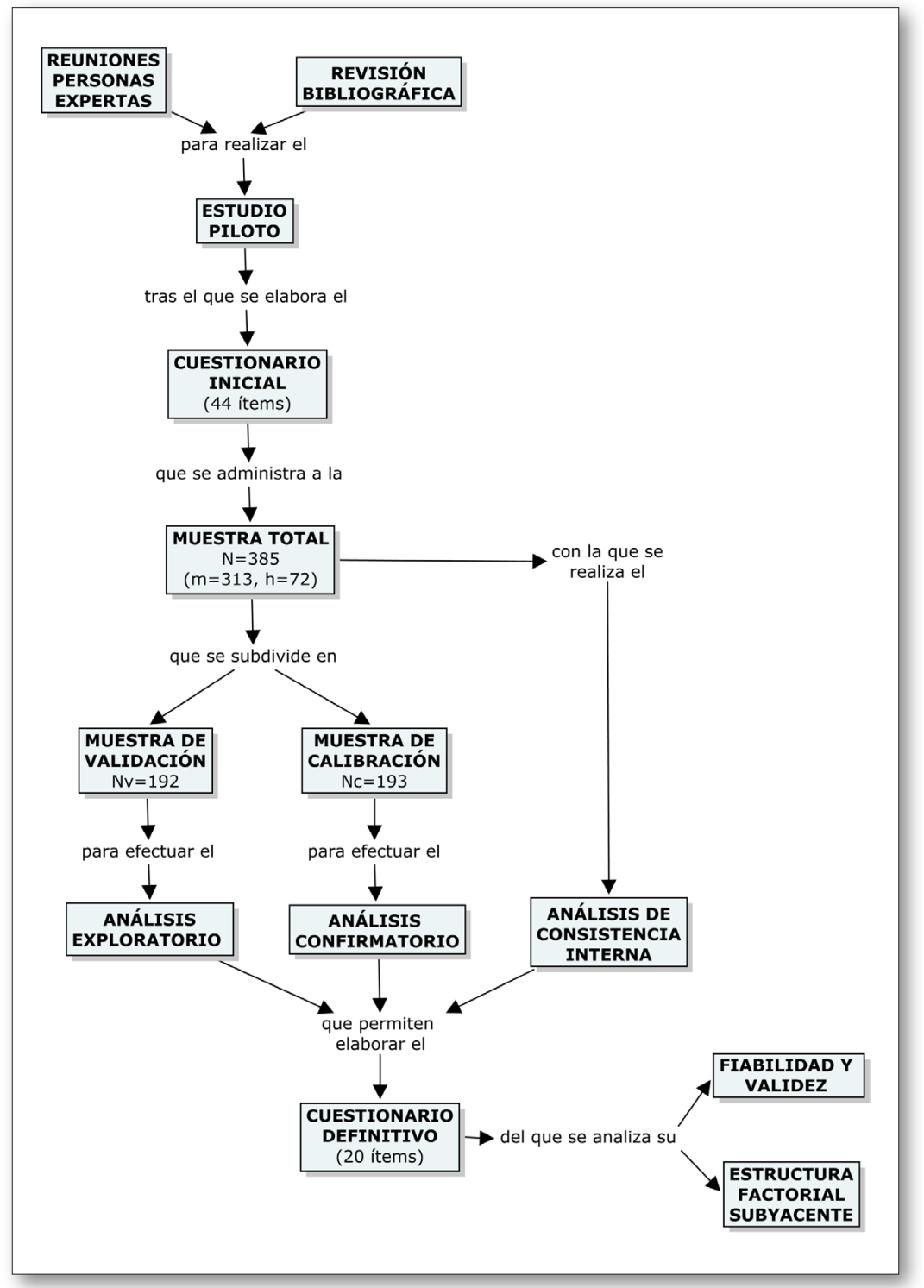

Fig. 1. Pasos seguidos en la validación del cuestionario. 


\section{Extracción de tipos docentes}

Para elaborar "tipos docentes» basados en las respuestas a los cuestionarios, se realiza un análisis clúster de las puntuaciones en los seis factores de la escala, con el fin de segmentar los casos en grupos de perfiles de respuestas similares.

\section{RESULTADOS}

Tras emplear el instrumento con el total de la muestra y valorar la fiabilidad y las posibles soluciones factoriales, se seleccionaron 20 ítems para la versión definitiva del instrumento (tabla 2).

\section{Análisis de fiabilidad}

Se muestra, a continuación, la tabla indicada (tabla 2), que muestra los estadísticos descriptivos y el análisis de la consistencia interna.

Tabla 2.

Estadísticos descriptivos y análisis de consistencia interna

\begin{tabular}{|c|c|c|c|c|c|c|c|}
\hline Ítem & Contenido del item & M & $D T$ & As & $K$ & $r$ & $\alpha_{\sin }$ \\
\hline i1 & La persona científica busca objetivamente la realidad. & 1,63 & 0,76 & 0,98 & 0,28 & .371 & .767 \\
\hline i2 & $\begin{array}{l}\text { El conocimiento científico se corresponde directamente con } \\
\text { la realidad. }\end{array}$ & 2,16 & 0,76 & 0,12 & $-0,50$ & .392 & .766 \\
\hline i3 & $\begin{array}{l}\text { Es necesario usar un método para descubrir y validar las } \\
\text { teorías. }\end{array}$ & 1,58 & 0,69 & 1,01 & 0,68 & .349 & .769 \\
\hline $\mathrm{i} 4$ & $\begin{array}{l}\text { La metodología científica garantiza la objetividad en el es- } \\
\text { tudio de la realidad. }\end{array}$ & 2,23 & 0,79 & 0,24 & $-0,32$ & .431 & .763 \\
\hline i5 & $\begin{array}{l}\text { La verdad científica se define como una descripción exacta } \\
\text { de la naturaleza. }\end{array}$ & 2,48 & 0,79 & 0,00 & $-0,42$ & .426 & .763 \\
\hline $\mathrm{i} 6$ & $\begin{array}{l}\text { La metodología científica es un proceso en etapas "paso a } \\
\text { paso». }\end{array}$ & 1,79 & 0,67 & 0,54 & 0,31 & .445 & .763 \\
\hline i7 & $\begin{array}{l}\text { La realización de investigaciones científicas tiene como fi- } \\
\text { nalidad resolver problemas de interés sobre el entorno que } \\
\text { nos rodea. }\end{array}$ & 1,86 & 0,67 & 0,53 & 0,60 & .357 & .769 \\
\hline i8 & $\begin{array}{l}\text { La ciencia es un conjunto de leyes y teorías que explican el } \\
\text { mundo. }\end{array}$ & 2,04 & 0,68 & 0,54 & 0,31 & .449 & .763 \\
\hline i9 & $\begin{array}{l}\text { A través de la experimentación, la persona investigadora } \\
\text { debe comprobar si su hipótesis es verdadera o falsa. }\end{array}$ & 1,37 & 0,54 & 1,12 & 0,24 & .341 & .770 \\
\hline $\mathrm{i} 10$ & $\begin{array}{l}\text { El conocimiento científico es generado y validado por con- } \\
\text { senso en la comunidad científica. }\end{array}$ & 2,11 & 0,70 & 0,21 & $-0,11$ & .353 & .769 \\
\hline i11 & Las leyes se validan por consenso de las personas científicas. & 2,41 & 0,78 & 0,09 & $-0,37$ & .355 & .768 \\
\hline i12 & $\begin{array}{l}\text { La ciencia evoluciona mediante la acumulación de teorías } \\
\text { verdaderas. }\end{array}$ & 1,97 & 0,84 & 0,37 & $-0,79$ & .462 & .760 \\
\hline i13 & $\begin{array}{l}\text { El progreso científico es descubrir teorías que se acerquen } \\
\text { cada vez más a la verdad. }\end{array}$ & 1,72 & 0,73 & 0,77 & 0,24 & .397 & .766 \\
\hline
\end{tabular}




\begin{tabular}{|c|c|c|c|c|c|c|c|}
\hline Item & Contenido del item & M & $D T$ & As & $K$ & $r$ & $\alpha_{s i n}$ \\
\hline i14 & $\begin{array}{l}\text { Existe un único método científico que siempre se acerca a } \\
\text { la verdad. }\end{array}$ & 3,26 & 0,82 & $-0,98$ & 0,39 & .129 & .784 \\
\hline i15 & $\begin{array}{l}\text { La ciencia es una actividad desarrollada por colectivos que } \\
\text { tienen ideas y técnicas comunes. }\end{array}$ & 2,77 & 0,77 & $-0,89$ & $-0,49$ & .268 & .774 \\
\hline i16 & $\begin{array}{l}\text { Es preciso diseñar y planificar una investigación científica } \\
\text { antes de comenzarla. }\end{array}$ & 1,34 & 0,60 & 1,89 & 3,81 & .288 & .773 \\
\hline i17 & $\begin{array}{l}\text { Toda investigación comienza por la observación sistemática } \\
\text { de un fenómeno. }\end{array}$ & 1,72 & 0,74 & 0,80 & 0,23 & .295 & .772 \\
\hline i18 & $\begin{array}{l}\text { Las afirmaciones de una persona científica siempre están } \\
\text { influenciadas por la comunidad científica y por las investi- } \\
\text { gaciones anteriores o precedentes. }\end{array}$ & 2,12 & 0,81 & 0,32 & $-0,40$ & .184 & .780 \\
\hline i19 & $\begin{array}{l}\text { Una teoría es desmentida si aparece un hecho que la con- } \\
\text { tradice. }\end{array}$ & 2,03 & 0,89 & 0,44 & $-0,66$ & .226 & .779 \\
\hline i20 & $\begin{array}{l}\text { Una persona científica evalúa sus afirmaciones acerca de la } \\
\text { ciencia exclusivamente a través de la evidencia contrastada. }\end{array}$ & 2,41 & 0,83 & 0,04 & $-0,57$ & .369 & .767 \\
\hline
\end{tabular}

$\mathrm{M}=$ media; DT = desviación típica; As = asimetría; $\mathrm{K}$ = curtosis; $\mathrm{r}$ = coeficiente de correlación entre el ítem y el resto de la escala; $\alpha_{\sin }=$ coeficiente alpha de Cronbach si el ítem fuese eliminado. El grado de acuerdo con los ítems manifestado va del 1 (máximo acuerdo) al 4 (mínimo acuerdo).

El coeficiente alfa de la escala es $\alpha=.778$, que indica que su consistencia interna es adecuada.

Para estudiar la consistencia interna de la escala, además de $\alpha$ también se calcularon los coeficientes $\Omega$ y glb. El coeficiente $\Omega$ se corresponde con el cuadrado de la correlación entre la puntuación en la escala y la variable latente común a todos los indicadores en el universo infinito de indicadores, de los que los de la escala son un subconjunto (McDonald, 1999). Por su parte, el glb (greatest lower bound, Woodhouse y Jackson, 1977) representa la mínima fiabilidad posible dada la matriz de covarianzas observadas, teniendo en cuenta la restricción de que la suma de las varianzas de los errores es máxima para los errores que no correlacionan con las otras variables (Ten Berge, Snijders y Zegers, 1981). En el caso del presente instrumento se calculó que glb $=.872$ y $\Omega=.777$.

\section{Análisis exploratorio}

La muestra original $(\mathrm{N}=385)$ se dividió en dos submuestras aleatorias (muestra de validación $\mathrm{N}_{\mathrm{V}}$ = 192 y de calibración $N_{C}=193$ ). Con la primera se realizó un análisis exploratorio para elaborar un modelo de las principales dimensiones implicadas y la segunda se utilizó para llevar a cabo un análisis factorial confirmatorio.

El análisis exploratorio se basa en determinar las correlaciones entre las variables en un conjunto de datos. Este tipo de análisis proporciona una estructura factorial, agrupando las variables que correlacionan entre sí.

Se realizó un análisis de componentes principales con rotación Promax, para que las cargas factoriales aparezcan más diferenciadas. La medida de adecuación muestral KMO es de .753 y la esfericidad de Bartlett tiene un nivel de significación de .000 , lo que apoya la pertinencia de proceder a la factorización de las variables.

Siguiendo el criterio estándar de Kaiser-Guttman (autovalores $>1$ ), a partir del análisis exploratorio se proponen 6 factores que explican el 56,8 \% de la varianza total. Sus autovalores y la varianza común que explican se presentan en la tabla 3. También se utilizó el programa Factor 9.3 para realizar la prueba MAP (Velicer, 1976) y el análisis paralelo basado en el análisis factorial de rango mínimo (Timmer- 
man y Lorenzo-Seva, 2011) sobre la matriz de correlaciones, que indicaron en ambos casos que debía retenerse solo un factor. Se tendrá en cuenta esta circunstancia al realizar el análisis confirmatorio, para valorar si la opción elegida (6 factores) es preferible a la opción de un único factor.

Tabla 3.

Estructura interna de la escala

\begin{tabular}{|l|c|c|c|c|}
\hline \multicolumn{1}{|c|}{ Dimensión } & Código & N. ${ }^{\text {items }}$ & Autovalor & \% varianza explicada \\
\hline Positivismo & POS & 5 & 4,33 & 21,7 \\
\hline Metaciencia & MTC & 4 & 1,75 & 8,7 \\
\hline Génesis del conocimiento científico & GEN & 4 & 1,50 & 7,5 \\
\hline Metodologías científicas & MTG & 2 & 1.36 & 6.8 \\
\hline Investigación & INV & 3 & 1,27 & 6,4 \\
\hline Validación de las teorías & VAL & 2 & 1,16 & 5,8 \\
\hline
\end{tabular}

En la tabla 4 se muestra la matriz de configuración. Los ítems de cada factor satisfacen los siguientes criterios:

- Saturación del ítem en el factor principal >.450.

- Saturación del ítem en los otros factores $<.400$.

- Diferencia de saturación entre el factor principal y el resto $>.200$.

Tabla 4

Matriz de configuración tras la realización del análisis de componentes principales y la rotación Promax. Se muestran solo las cargas factoriales mayores de .300, y se resaltan en negrita los mayores de .400 .

Test de esfericidad $=.000 ; \mathrm{KMO}=.753$.

\begin{tabular}{|c|c|c|c|c|c|c|c|}
\hline \multirow{2}{*}{ Item } & \multirow[t]{2}{*}{ Contenido del item } & \multicolumn{6}{|c|}{ Factores } \\
\hline & & POS & MTC & GEN & MTG & INV & VAL \\
\hline i1 & La persona científica busca objetivamente la realidad. & .748 & & & & & \\
\hline i2 & $\begin{array}{l}\text { El conocimiento científico se corresponde directamente } \\
\text { con la realidad. }\end{array}$ & .722 & & & & & \\
\hline i3 & $\begin{array}{l}\text { Es necesario usar un método para descubrir y validar las } \\
\text { teorías. }\end{array}$ & .659 & & & & & \\
\hline i4 & $\begin{array}{l}\text { La metodología científica garantiza la objetividad en el es- } \\
\text { tudio de la realidad. }\end{array}$ & .579 & .369 & & & & \\
\hline i5 & $\begin{array}{l}\text { La verdad científica se define como una descripción exacta } \\
\text { de la naturaleza. }\end{array}$ & .502 & & & & & \\
\hline i6 & $\begin{array}{l}\text { La metodología científica es un proceso en etapas "paso a } \\
\text { paso". }\end{array}$ & & .771 & & & & \\
\hline i7 & $\begin{array}{l}\text { La realización de investigaciones científicas tiene como fi- } \\
\text { nalidad resolver problemas de interés sobre el entorno que } \\
\text { nos rodea. }\end{array}$ & & .663 & & & & -.330 \\
\hline i8 & $\begin{array}{l}\text { La ciencia es un conjunto de leyes y teorías que explican } \\
\text { el mundo. }\end{array}$ & & .659 & & & & \\
\hline i9 & $\begin{array}{l}\text { A través de la experimentación, la persona investigadora } \\
\text { debe comprobar si su hipótesis es verdadera o falsa. }\end{array}$ & & .626 & & -.377 & & \\
\hline
\end{tabular}




\begin{tabular}{|c|c|c|c|c|c|c|c|}
\hline \multirow{2}{*}{ Item } & \multirow[t]{2}{*}{ Contenido del item } & \multicolumn{6}{|c|}{ Factores } \\
\hline & & POS & MTC & GEN & MTG & INV & VAL \\
\hline i 10 & $\begin{array}{l}\text { El conocimiento científico es generado y validado por con- } \\
\text { senso en la comunidad científica. }\end{array}$ & & & .853 & & & \\
\hline i11 & Las leyes se validan por consenso de las personas científicas. & & & .724 & & & \\
\hline i12 & $\begin{array}{l}\text { La ciencia evoluciona mediante la acumulación de teorías } \\
\text { verdaderas. }\end{array}$ & & & .636 & & & \\
\hline i13 & $\begin{array}{l}\text { El progreso científico es descubrir teorías que se acerquen } \\
\text { cada vez más a la verdad. }\end{array}$ & & & .463 & & & \\
\hline i14 & $\begin{array}{l}\text { Existe un único método científico que siempre se acerca a } \\
\text { la verdad. }\end{array}$ & & & & .873 & & \\
\hline i15 & $\begin{array}{l}\text { La ciencia es una actividad desarrollada por colectivos que } \\
\text { tienen ideas y técnicas comunes. }\end{array}$ & & & & .699 & & \\
\hline i16 & $\begin{array}{l}\text { Es preciso diseñar y planificar una investigación científica } \\
\text { antes de comenzarla. }\end{array}$ & .317 & & & & .683 & \\
\hline i17 & $\begin{array}{l}\text { Toda investigación comienza por la observación sistemática } \\
\text { de un fenómeno. }\end{array}$ & & .391 & & & .608 & \\
\hline i18 & $\begin{array}{l}\text { Las afirmaciones de una persona científica siempre están } \\
\text { influenciadas por la comunidad científica y por las investi- } \\
\text { gaciones anteriores o precedentes. }\end{array}$ & & & & & .570 & \\
\hline i19 & $\begin{array}{l}\text { Una teoría es desmentida si aparece un hecho que la con- } \\
\text { tradice. }\end{array}$ & & & & & & .785 \\
\hline i20 & $\begin{array}{l}\text { Una persona científica evalúa sus afirmaciones acerca de la } \\
\text { ciencia exclusivamente a través de la evidencia contrastada. }\end{array}$ & & & & & & .536 \\
\hline
\end{tabular}

Por tanto, los resultados del análisis exploratorio muestran una estructura factorial con seis factores bien definidos.

- El primer factor (POS Positivismo) está formado por 5 ítems y explica el 21,7 \% da varianza. Incluye ítems relacionados con la objetividad de la ciencia y la descripción exacta de la fenomenología como una realidad palpable dependiente o independiente del paradigma de observación.

- El segundo factor (MTC - Metaciencia) está formado por 4 ítems que explican el 8,7 \% de la varianza común. Sus ítems hacen referencia a la visión algorítmica u holística de la ciencia al integrar heurísticos asociados.

- El tercer factor (GEN - Génesis del conocimiento científico) se compone de 4 ítems que explican el 7,5 \% de la varianza. Explicitan la acumulación de conocimiento científico objetivo en gradiente hacia una verdad focal versus a través del consenso intersubjetivo de la comunidad científica.

- El cuarto factor (MTG - Metodologías científicas) lo forman 2 ítems que explican el 6,8 \% de la varianza común. Se refieren a la existencia de un único método científico o de un pluralismo metodológico.

- El quinto factor (INV - Investigación) está compuesto por 3 ítems que explican el 6,4 \% de la varianza. Estos ítems hacen referencia a cómo se desarrolla la investigación científica en el imaginario colectivo, si de forma inductiva o hipotético-deductiva.

- Por último, el sexto factor (VAL - Validación de las teorías) lo forman 2 ítems que explican el $5,8 \%$ de varianza común y que indican validación a través de evidencias contrastadas versus desmentido por hechos contradictorios singulares. 
La correlación entre estos factores puede verse en la tabla 5.

Tabla 5.

Matriz de correlaciones entre factores

\begin{tabular}{|c|r|r|r|r|r|r|}
\hline Componente & \multicolumn{1}{|c|}{ POS } & MTC & \multicolumn{1}{|c|}{ GEN } & \multicolumn{1}{c|}{ MTG } & \multicolumn{1}{c|}{ INV } & VAL \\
\hline POS & 1,000 &, 375 &, 280 &, 061 &,- 026 &, 195 \\
MTC &, 375 & 1,000 &, 404 &, 152 &, 047 &, 247 \\
GEN &, 280 &, 404 & 1,000 &, 189 &, 033 &, 187 \\
MTG &, 061 &, 152 &, 189 & 1,000 &,- 023 &, 005 \\
INV &,- 026 &, 047 &, 033 &,- 023 & 1,000 &,- 044 \\
VAL &, 195 &, 247 &, 187 &, 005 &,- 044 & 1,000 \\
\hline
\end{tabular}

\section{Análisis confirmatorio}

El análisis confirmatorio es el siguiente paso tras la realización del análisis exploratorio para determinar la estructura factorial del conjunto de datos. Una vez que se ha explorado dicha estructura, se confirma mediante este tipo de análisis.

Se empleó la segunda submuestra $\left(\mathrm{N}_{\mathrm{C}}=193\right)$ para llevar a cabo el análisis factorial confirmatorio. Este análisis se realizó con el software AMOS 21, utilizando el método de estimación de máxima verosimilitud. Se consideraron tres modelos:

- M1: un solo factor, como indicaba el resultado del análisis paralelo y de la prueba MAP.

- M2: seis factores no relacionados.

- M3: seis factores relacionados entre sí.

Se recomienda emplear varios índices de ajuste para valorar los modelos (Markland, 2007; Hooper, Coughlan y Mullen, 2008). Así, aquí se utiliza una combinación de índices de ajuste absolutos y relativos (tabla 6).

Tabla 6.

Índices de ajuste de los modelos. Submuestra $2\left(\mathrm{~N}_{2}=193\right)$

\begin{tabular}{|c|l|c|c|c|c|c|c|c|c|}
\hline \multicolumn{2}{|c|}{ Modelo $\chi^{2}$} & \multicolumn{9}{c|}{ Índices de ajuste } \\
\cline { 3 - 10 } & $\mathrm{p}$ & $\chi^{2} / \mathrm{gl}$ & AIC & CFI & \multicolumn{3}{c|}{ RMSEA 90 \% CI } & \\
\hline M1 & Único factor & 267,76 & .000 & 1,58 & 387.76 & .779 & .055 & {$[.042$} & $.067]$ \\
\hline M2 & $\begin{array}{l}\text { Seis factores no } \\
\text { relacionados }\end{array}$ & 355,31 & .000 & 2,06 & 475.31 & .585 & .075 & {$[.064$} & $.085]$ \\
\hline M3 & $\begin{array}{l}\text { Seis factores } \\
\text { relacionados }\end{array}$ & 194,44 & .017 & 1,25 & 344.44 & .911 & .036 & {$[.016$} & $.052]$ \\
\hline
\end{tabular}

- En los tres modelos la prueba $\chi^{2}$ es significativa, lo que apuntaría a que los modelos teóricos no se ajustan adecuadamente a los datos. No obstante, el resultado de esta prueba se ve afectado por diferentes factores, entre ellos el tamaño de la muestra, siendo muy difícil obtener un resultado no significativo cuando este es grande (Jöreskog y Sörbom, 1993).

- El valor de $\chi^{2}$ normado, que se utiliza para reducir la sensibilidad de $\chi^{2}$ al tamańo de la muestra dividiéndolo entre los grados de libertad, se considera perfecto si su valor es 1 . No hay consenso 
acerca de cuál sería el máximo valor aceptable para este heurístico, considerándose correctos desde valores menores de 2 como criterio más estricto (Tabachnick y Fidell, 2007) hasta los menores de 5 como criterio más laxo (Hu y Bentler, 1999). Empleando este heurístico, M1 y M3 tendrían un buen ajuste, siendo algo mejor el segundo de ellos $\left(\chi_{\mathrm{M} 1}^{2}=1,58 ; \chi_{\mathrm{M} 3}^{2}=1,25\right)$. M2 no superaría el criterio más estricto $\left(\chi_{\mathrm{M} 2}^{2}=2,06\right)$.

- El criterio de información de Akaike (AIC) sirve para estimar la calidad relativa de un modelo con respecto a otros, y se basa en la cantidad relativa de información que se pierde al emplear un modelo dado (Akaike, 1974). Los valores más pequeños indican un mejor ajuste y un modelo más parsimonioso. En el caso de los modelos estudiados, AIC es menor en M3, lo que apunta a que es el modelo con mejor bondad de ajuste de los tres. El segundo mejor modelo en este sentido sería M1, seguido por M2.

- Para el cálculo del índice CFI se asume que todas las variables latentes no están correlacionadas y se compara la matriz de covarianzas de la muestra con este modelo nulo. Toma valores entre 0 y 1 , indicando un mejor ajuste cuanto mayor sea su valor. Bentler (1990) indica que un valor superior a .90 es aceptable, y Hu y Bentler (1999) recomiendan para garantizar un ajuste superior un valor mayor que .95. El valor de CFI calculado para $\mathrm{M} 3$ es aceptable $\left(\mathrm{CFI}_{\mathrm{M} 3}=.911\right)$, mientras que no lo es para los modelos $\mathrm{M} 1\left(\mathrm{CFI}_{\mathrm{M} 1}=.779\right)$ y $\mathrm{M} 2\left(\mathrm{CFI}_{\mathrm{M} 2}=.585\right)$.

- El índice RMSEA refleja la adecuación del modelo, con parámetros desconocidos pero estimados de manera óptima, a la matriz de covarianzas de la población (Byrne, 1998). Favorece modelos parsimoniosos. Hu y Bentler (1999) proponen como valores buenos los inferiores a .05 y aceptables los menores de .10. Utilizando este criterio el valor de RMSEA es aceptable en M1 $\left(\right.$ RMSEA $\left._{\mathrm{M} 1}=.055\right)$ y M2 (RMSEA $\left.{ }_{\mathrm{M} 1}=.075\right)$, y bueno en M3 RMSEA $\left._{\mathrm{M} 3}=.036\right)$.

Todo lo antedicho indica que el mejor modelo de los presentados es el de 6 factores interrelacionados, por lo que fue el que se decidió retener (figura 2).

\section{Evidencias de validez}

- Validez de contenido: la adecuación de los ítems seleccionados al dominio de interés, así como la representatividad y corrección técnica de los ítems se sustenta en la revisión bibliográfica y en el trabajo realizado en las reuniones de personas expertas.

- Validez convergente: para su estudio se analizaron los coeficientes de regresión de las dimensiones en la predicción de las puntuaciones de los ítems que los componen (Hair, Black, Babin y Anderson, 2010). En todos los casos son significativamente diferentes de cero ( $\mathrm{p}<.05)$, lo que indica que los ítems de cada factor comparten una proporción significativa de varianza común. En este sentido, siete ítems tienen cargas factoriales superiores a .700, y solo un ítem no alcanza el valor de .500 recomendado por Hair et al. (2010).

- Validez discriminante: la inexistencia de saturaciones cruzadas en la estructura factorial propuesta es una evidencia de validez discriminante, ya que esta implica que los ítems individuales forman parte de un único factor. 


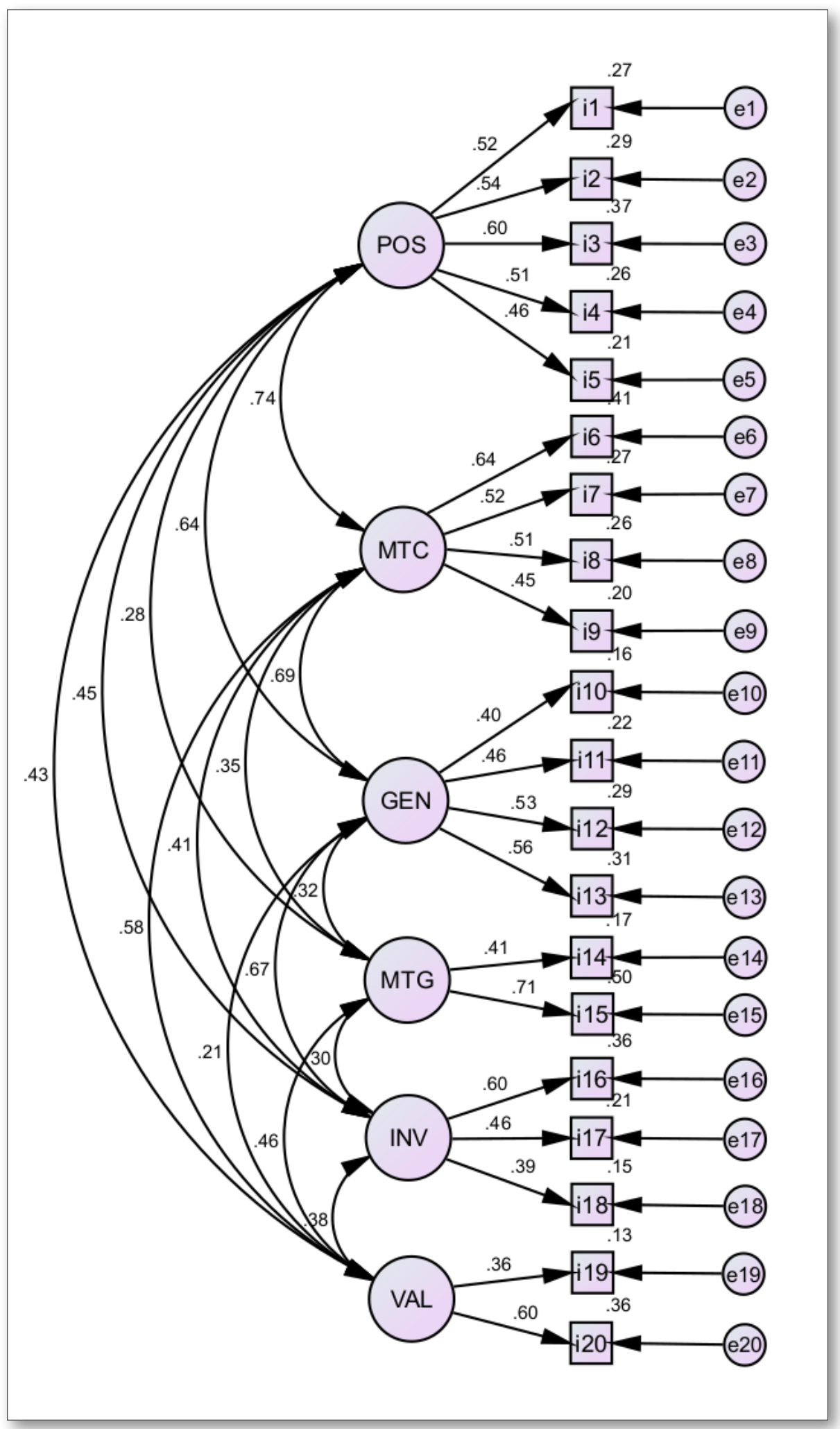

Fig. 2. Análisis factorial confirmatorio $\left(\mathrm{N}_{\mathrm{v}}=193\right) \cdot \chi^{2}=194,44 ; \mathrm{p}<.017 ; \chi^{2} / \mathrm{gl}=1,25 ; \mathrm{AIC}=344,44$; $\mathrm{CFI}=.911 ; \mathrm{RMSEA}=.036[.016 .022]$. 


\section{Tipos docentes}

Para elaborar «tipos docentes» basados en las respuestas a los cuestionarios, se realiza un análisis clúster de las puntuaciones en los seis factores de la escala, con el fin de segmentar los casos en grupos de perfiles de respuestas similares. Se emplea la muestra total $(\mathrm{N}=385)$. El análisis constó de los siguientes pasos:

- Cálculo de las puntuaciones en los factores de la escala.

- Comprobación de variables y supuestos del análisis clúster.

- Obtención de grupos mediante análisis jerárquico y valoración del ajuste conjunto.

- Análisis clúster no jerárquico (K-Medias) para ajuste fino.

- Validación de las soluciones clúster.

En la figura 3 se muestra la solución clúster propuesta. Como se puede apreciar, se diferencian tres tipos docentes (1, 2 y 3 ). En la tabla 7 se muestran descriptivos de dichos conglomerados.

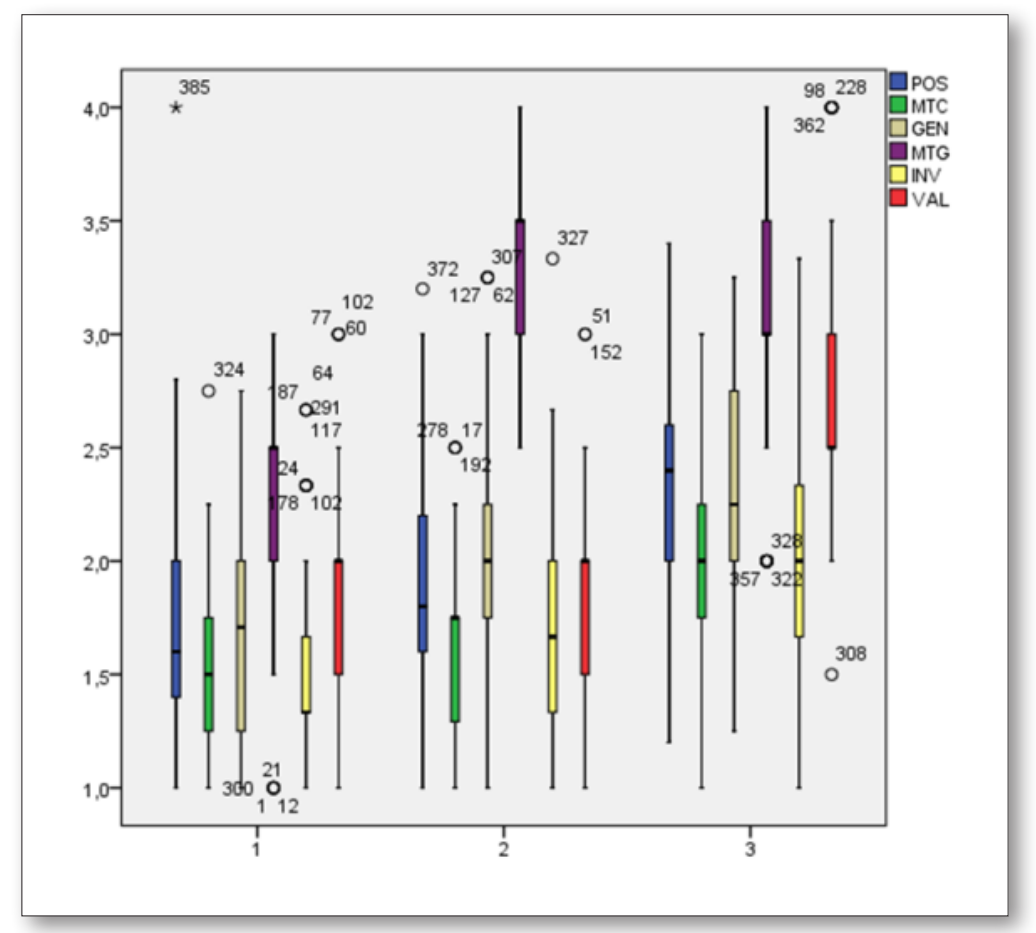

Fig. 3. Tipos docentes 1,2 y 3 propuestos.

Tabla 7.

Descriptivos de los tipos docentes propuestos

\begin{tabular}{|c|c|c|c|c|c|c|c|}
\hline Tipo docente & POS & MTC & GEN & MTG & INV & VAL & Tamaño del clúster \\
\hline 1 & 1,71 & 1,52 & 1,69 & 2,26 & 1,51 & 1,79 & 94 \\
\hline 2 & 1,89 & 1,62 & 1,96 & 3,41 & 1,63 & 1,88 & 132 \\
\hline 3 & 2,30 & 2,03 & 2,34 & 3,13 & 1,94 & 2,76 & 159 \\
\hline
\end{tabular}


Se pueden caracterizar estos tipos docentes de la siguiente manera:

- Tipo docente 1: clúster formado por personas con puntuaciones en los factores que denotan una clara tendencia al positivismo, una visión algorítmica de la ciencia con un único método inductivo que avanza acumulativamente hacia la verdad, validando sus resultados solo a través de la evidencia.

- Tipo docente 2: muy similar al anterior, pero con puntuaciones algo más elevadas en todos los factores. Destaca que una de dichas puntuaciones sí es mucho mayor que en el caso del tipo docente 1: el profesorado en formación de este clúster admite en mucha mayor medida un pluralismo metodológico.

- Tipo docente 3: sus puntuaciones son en general cercanas a las del punto medio de la escala Likert. Las ideas sobre la ciencia de las personas que forman este conglomerado son, por tanto, menos ingenuas y conciben en mayor medida la ciencia como una actividad humana. Con todo, sus puntuaciones no son elevadas.

\section{DISCUSIÓN Y CONCLUSIONES}

Se ha presentado el proceso de diseño y validación de un cuestionario para la indagación de las concepciones sobre la NOS y se han expuesto diferentes indicadores de su fiabilidad y de su validez de contenido, convergente y discriminante. Los resultados del análisis exploratorio muestran una estructura factorial con seis factores bien definidos. El análisis confirmatorio indica que dicho modelo con los seis factores relacionados entre sí tiene un buen ajuste, el cual es mejor que el de los otros modelos considerados.

Por todo ello, se concluye que el cuestionario tiene una fiabilidad y validez correctas y, en consecuencia, se considera un instrumento útil y se propone su uso para continuar con la investigación. En este sentido, el fin último de la línea de estudio que nos ocupa es indagar sobre las creencias acerca de la ciencia del profesorado en formación, a fin de mejorar los procesos de formación inicial. ¿`Son sus creencias informadas, parcialmente informadas o ingenuas? ¿Qué piensa sobre qué es y qué no es ciencia, o cómo se trabaja en ciencia?

Conviene destacar que los factores hallados al validar el instrumento son relevantes en este contexto, ya que proporcionan indicadores sobre el positivismo, las creencias sobre la generación del pensamiento científico, las metodologías, etc., aspectos todos ellos de relevancia y relacionados con la NOS. De esta manera, los tipos docentes hallados al realizar el análisis clúster se pueden encuadrar en las creencias ingenuas (tipos docentes 1 y 2) y en las parcialmente informadas (tipo docente 3). Es importante subrayar la no aparición de un clúster que se corresponda con las creencias informadas (Schwartz y Lederman, 2008). En todo caso, conviene destacar el carácter exploratorio y fundamentalmente ateórico del análisis clúster, por lo que la pretensión de este estudio no va más allá de formular propuestas fundamentadas que puedan ser de interés para la investigación futura.

En estudios posteriores se pretende proporcionar resultados descriptivos detallados de las puntuaciones en los diferentes factores y realizar análisis estadísticos inferenciales para valorar la existencia de diferencias por sexo, titulación, edad y otras variables de interés. Otra posible utilidad es emplear el cuestionario antes y después de una intervención educativa sostenida, para valorar si esta ha contribuido a la evolución de las ideas del colectivo investigado (Izquierdo, García, Quintanilla y Adúriz, 2016). Como limitaciones del estudio conviene señalar que, aunque el tamaño de la muestra es suficiente, sería de interés realizar un nuevo estudio con una muestra mayor; también se puede apuntar que se han extraído factores compuestos por pocos ítems, lo cual en general es preferible evitar, salvo que aparezcan con claridad y sean de relevancia en el contexto del estudio, como aquí acontece. Asimismo, 
las evidencias de fiabilidad y validez expuestas son correctas, pero podrían intentar mejorarse en nuevas versiones del instrumento partiendo del trabajo aquí realizado y atendiendo a las consideraciones expuestas. Se utilizarán, también, metodologías cualitativas (grupos de discusión, entrevistas y estudios de caso) a fin de indagar acerca de los significados que el profesorado en formación inicial asocia a sus respuestas al cuestionario.

Otro aspecto susceptible de mejora es la elaboración de un cuestionario nuevo que aborde, de manera más específica, las concepciones sobre las relaciones entre ciencia, tecnología y sociedad en la línea de la tecnociencia contemporánea (Echeverría y Martínez, 2009; Park, Wu y Erduran, 2020).

Los aspectos estudiados tienen una innegable repercusión en la planificación de la formación universitaria del profesorado; por tanto, es absolutamente necesario investigar su influencia para incidir en la mejora de la oferta formativa de la Universidad, en el diseño de los currículos, de las metodologías docentes y en la selección y utilización de recursos didácticos (Bonil y Márquez, 2011; Izquierdo, 2007; Márquez y Artés, 2016; Pujol, 2008; Sanmartí, 2011; Vílchez y Bravo, 2015).

La pregunta que surge de inmediato es la siguiente: ¿Es posible modificar esas concepciones? Y si lo es, ¿cómo se puede llevar a cabo este cambio? La cuestión ha sido objeto de atención en la investigación en didáctica de las ciencias experimentales y existen diversas propuestas. Porlán, Rivero y Martín (1997) sostienen que esta evolución es un proceso de reestructuración y construcción de nuevos significados, basado en la interacción y el contraste con otras ideas y experiencias. Por su parte, AdúrizBravo e Izquierdo-Aymerich (2002) sostienen que a la hora de diseñar actividades didácticas sobre NOS existen rasgos metodológicos que se deben tener en cuenta, como situar el lenguaje en el centro de dichas actividades, utilizar materiales variados (textos literarios, episodios históricos, juegos o documentales) y reflexionar sobre el conocimiento científico (contenidos, métodos, valores o evolución).

Por nuestra parte, ańadiremos que la didáctica de las ciencias experimentales no puede realizar este proceso en solitario, algo que se olvida con más frecuencia de la que sería deseable, sino que es necesaria la participación de todo el equipo docente.

\section{AGRADECIMIENTOS}

Proyectos de investigación financiados por el Ministerio de Ciencia, Innovación y Universidades EDU2017-82915-R y por FEDER/Ministerio de Ciencia, Innovación y Universidades - Agencia Estatal de Investigación/ Proyecto ESPIGA («Promoviendo el Desarrollo del Pensamiento Crítico y de las dimensiones de Implicación Cognitiva y Emocional de los desempeños Epistémicos en las Clases de Ciencias en la Era de la Posverdad»), referencia PGC2018-096581-B-C22.

\section{REFERENCIAS BIBLIOGRÁFICAS}

Abd-El-Khalick, F. y Akerson, V. L. (2009). The influence of metacognitive training on preservice elementary teachers' conceptions of nature of science. International Journal of Science Education, $31,2161-2184$. https://doi.org/10.1080/09500690802563324

Adúriz-Bravo, A. (2005). Una introducción a la naturaleza de la ciencia: La epistemología en la enseñan$z a$ de las ciencias naturales. Buenos Aires: Fondo de Cultura Económica.

Adúriz-Bravo, A. (2006). La epistemología en la formación de profesores de ciencias. Educación y Pedagogía, 18(45), 25-36. 
Adúriz-Bravo, A. (2008). Áreas de investigación en la didáctica de las ciencias experimentales: La naturaleza de la ciencia. En C. Merino, A. Gómez y A. Adúriz-Bravo (Coords.), Áreas y estrategias de investigación en la didáctica de las ciencias experimentales (pp. 111-125). Bellaterra: Servei de Publicacions de la UAB.

Adúriz-Bravo, A. e Izquierdo-Aymerich, M. (2002). Directrices para la formación epistemológica del futuro profesorado de ciencias naturales. En G. A. Perafán y A. Adúriz-Bravo (Comps.), Pensamiento y conocimiento de los profesores: Debate y perspectivas internacionales (pp. 127-139). Bogotá: Universidad Pedagógica Nacional.

Akaike, H. (1974). A New Look at the Statistical Model Identification. IEE Transactions on Automatic Control, 19(6), 716-723.

https://doi.org/10.1109/TAC.1974.1100705

Álvarez Lires, M., Arias, A., Pérez Rodríguez, U. y Serrallé, J. F. (2013). La historia de las ciencias en el desarrollo de competencias científicas. Enseñanza de las Ciencias, 31(1), 213-233.

Angulo, F. (2002). Aprender a enseñar ciencias: análisis de una propuesta para la formación inicial del profesorado de secundaria, basada en la metacognición (Tesis de doctorado inédita). Bellaterra (Barcelona): Universitat Autònoma de Barcelona.

Arias Correa, A. (2012). Implicacións curriculares e didácticas no ensino das ciencias das concepcións sobre a ciencia e a metodoloxía en alumnado de Maxisterio: o traballo por proxectos (Tesis de doctorado inédita). Vigo: Universidade de Vigo.

Bachelard, G. (1938). La formation de l'esprit scientifique. París: Vrin.

Bentler, P. M. (1990). Comparative Fit Indexes in Structural Models. Psychological Bulletin, 107(2), 238-246.

https://doi.org/10.1037/0033-2909.107.2.238

Blanco, Á., Sanz, B. y Vallejo, P. M. (2003). Construcciones de escalas de actitudes tipo Likert. Madrid: Editorial La Muralla.

Bonil, J. y Márquez, C. (2011). ¿Qué experiencias manifiestan los futuros maestros sobre las clases de ciencias? Implicaciones para su formación. Revista de Educación, 354, 447-472. https://doi.org/10.4438/1988-592X-RE-2011-354-007

Brandão, R. V. (2008). Investigando a aprendizagem do campo conceitual associado à modelagem cientifca por parte de professores de física do ensino médio (Tesis de máster inédita). Porto Alegre: Instituto de Física, Universidade Federal do Rio Grande do Sul.

Brandão, R. V., Araujo, I. S., Veit, E. A. y Lang, F. S. (2011). Validación de un cuestionario para investigar concepciones de profesores sobre ciencia y modelado científico en el contexto de la física. Revista Electrónica de Investigación en Educación en Ciencias, 1(6), 43-60.

Briceño, J. J. y Benarroch, A. (2009). Concepciones y creencias sobre ciencia, aprendizaje y enseñanza de profesores universitarios de ciencias. Revista Electrónica de Investigación en Educación en Ciencias, $8(1), 24-41$.

Byrne, B. M. (1998). Structural Equation Modeling with LISREL, PRELIS and SIMPLIS: Basic Concepts, Applications and Programming. Mahwah, Nueva Jersey: Lawrence Erlbaum Associates.

Claxton, G. (1994). Educar mentes curiosas. Madrid: Visor.

Clough, M. P. (2006). Learners' responses to the demands of conceptual change: Considerations for effective nature of science instruction. Science \& Education, 15(5), 463-494. https://doi.org/10.1007/s11191-005-4846-7

Cummins, R. y Gullone, E. (2000). Why we should not use 5-point Likert scales: The case for subjective quality of life measurement. En Proceedings of Second international conference on quality of life in cities (pp. 74-93). 
Driver, R., Leach, J., Millar, R. y Scott, P. (1996). Young peoples's images of science. Buckingham, UK: Open University Press.

Duit, R. (2009). Bibliography - STCSE Students' and Teachers' Conceptions and Science Education. Obtenido de https://www.if.ufrj.br/ marta/aprendizagememfisica/fv09.pdf

Duit, R., Gropengieber, H. y Kattmann, U. (2005). Towards science education research that is relevant for improving practice: The model of educational reconstruction. En H. Fischer (Ed.), Developing Standards in Research on Science Education (pp. 1-9). Londres: Taylor \& Francis Group.

Echeverría, J. y González, M. (2009). La teoría del actor-red y la tesis de la tecnociencia. Arbor, 738, 705-720. https://doi.org/10.3989/arbor.2009.738n1047

Ferreira-Gauchía, C., Vilches, A. y Gil-Pérez, D. (2012). Concepciones acerca de la naturaleza de la tecnología y de las relaciones Ciencia, Tecnología, Sociedad y Ambiente en la educación tecnológica. Enseñanza de las Ciencias: Revista de Investigación y Experiencias Didácticas, 30(2), 197-218.

Furió, C., Solbes, J. y Carrascosa, J. (2004). Las ideas alternativas sobre conceptos científicos: tres décadas de investigación. resultados y perspectivas. Alambique, 48, 64-78.

Hair, F., Black, W. C., Babin, B. J. y Anderson, R. E. (2010). Multivariate data analysis. Nueva Jersey: Prentice-Hall.

Hooper, D., Coughlan, J. y Mullen, M. R. (2008). Structural Equation Modelling: Guidelines for Determining Model Fit. Electronic Journal of Business Research Methods, 6(1), 53-60. https://doi.org/10.21427/d7cf7r

$\mathrm{Hu}, \mathrm{L} . \mathrm{y}$ Bentler, P. M. (1999). Cutoff criteria for fit indexes in covariance structure analysis: Conventional criteria versus new alternatives. Structural Equation Modelling, 6, 1-55. https://doi.org/10.1080/10705519909540118

Izquierdo, M. (2007). Enseñar ciencias, una nueva ciencia. Enseñanza de las Ciencias Sociales, 6, 125138.

Izquierdo, M., García, A., Quintanilla, M. y Adúriz, A. (2016). Historia, filosofía y didáctica de las ciencias: aportes para la formación del profesorado de ciencias. Bogotá: Universidad Distrital.

Jöreskog, K. y Sörbom, D. (1993). LISREL 8: Structural Equation Modeling with the SIMPLIS Command Language. Chicago, IL: Scientific Software International Inc.

Justi, R. S. y Gilbert, J. K. (2002). Modelling, teachers' views on the nature of modelling, and implications for the education of modellers. International Journal of Science Education, 24(4), 369-387. https://doi.org/10.1080/09500690110110142

Lederman, N. G. (2007). Nature of science: past, presente and future. En S. Abell y N. G. Lederman (Eds.), Handbook of Research on Science Education (pp. 831-880). Nueva Jersey: Lawrence Erlbaum Associates Publishers.

Lederman, N. G. (2018). La siempre cambiante contextualización de la naturaleza de la ciencia: documentos recientes sobre la reforma de la educación científica en los Estados Unidos y su impacto en el logro de la alfabetización científica. Enseñanza de las Ciencias, 36(2), 5-22. https://doi.org/10.5565/rev/ensciencias.2661

Lederman, N. G., Abd-El-Khalick, F., Bell, R. L. y Schwartz, R. S. (2002). Views of nature of science questionnaire: Toward valid and meaningful assessment of learners' conceptions of nature of science. Journal of Research in Science Teaching, 39(6), 497-521. https://doi.org/10.1002/tea.10034

Lederman, N. G. y Niess, M. L. (1997). The nature of science: Naturally? School Science and Mathematics, 97(1), 1-2. 
Markland, D. (2007). The golden rule is that there are no golden rules: A commentary on Paul Barrett's recommendations for reporting model fit in structural equation modelling. Personality and Individual Differences, 42, 851-858. https://doi.org/10.1016/j.paid.2006.09.023

Márquez, C. y Artés, M. (2016). Enseñanza de las Ciencias de la Tierra: Revista de la Asociación Española para la Enseñanza de las Ciencias de la Tierra, 24(2), 169-181.

Martín, C., Prieto, T. y Jiménez, M. A. (2015). Tendencias del profesorado de ciencias en formación inicial sobre las estrategias metodológicas en la enseńanza de las ciencias. Estudio de un caso en Málaga. Enseñanza de las Ciencias, 33(1), 167-184. https://doi.org/10.5565/rev/ensciencias. 1500

McComas, W. F., Clough, M. P. y Almazroa, H. (1998). The role and character of the nature of science in science education. En W. F. McComas (Ed.), The nature of science in science education: Rationales and strategies (pp. 3-39). Dordrecht: Kluwer.

McDonald, R. P. (1999). Test theory: A unified treatment. Mahwah, NJ: Lawrence Erlbaum.

Park, W., Wu, J. y Erduran, S. (2020). The Nature of STEM Disciplines in the Science Education Standards Documents from the USA, Korea and Taiwan. Sci \& Educ https://doi.org/10.1007/s11191-020-00139-1

Pope, M. y Gilbert, J. (1983). Personal Experience and the Construction of Knowledge in Science. Science Education, 67(2), 193-203. https://doi.org/10.1002/sce.3730670208

Porlán, R., Díaz, J. E. G. y De León, P. C. (1988). Constructivismo y enseñanza de las ciencias. Madrid: Díada Editora.

Porlán, R., Rivero, A. y Martín, R. (1997). Conocimiento profesional y epistemología de los profesores I: Teoría, métodos e instrumentos. Enseñanza de las Ciencias, 15(2), 155-171.

Pujol, R. M. (2008). Pensar en la escuela primaria para pensar en la formación de su profesorado, desde la DCE, en el marco del nuevo grado. Ponencia presentada en XXIII Encuentros de Didáctica de las Ciencias Experimentales. Almería, España.

Ravanal, E., Joglar, C., Quintanilla, M. y Labarrere, A. (2009). Noción sobre enseñanza de las ciencias en profesores de biología en activo y sus implicancias en el desarrollo de competencias de pensamiento científico. Ponencia presentada en el VII Encontro Nacional de Pesquisa em Educaçâo em Ciências. Florianópolis, Brasil.

Sanmartí, N. (2011). Evaluar para aprender, evaluar para calificar. En P. Cañal (Coord.), Didáctica de la Biología y la Geología (pp. 151-174). Barcelona: Graó.

Schwartz, R. y Lederman, N. (2008). What scientists say: scientists views of Nature of Science and relation to science context. International Journal of Science Education, 30(6), 727-771. https://doi.org/10.1080/09500690701225801

Serrallé, J. F. (2018). A Historia das Ciencias no desenvolvemento de competencias cientificas: unha perspectiva socioconstrutivista e de xénero (Tesis doctoral inédita). Vigo: Universidade de Vigo.

Tabachnick, B. G. y Fidell, L. S. (2007). Using Multivariate Statistics. Nueva York: Allyn and Bacon.

Ten Berge, J. M. F., Snijders, T. A. B. y Zegers, F. E. (1981). Computational aspects of the greatest lower bound to reliability and constrained minimum trace factor analysis. Psychometrika, 46, 201-213. https://doi.org/10.1007/BF02293900

Timmerman, M. E. y Lorenzo-Seva, U. (2011). Dimensionality Assessment of Ordered Polytomous Items with Parallel Analysis. Psychological Methods, 16, 209-220.

https://doi.org/10.1037/a0023353 
Velicer, W. F. (1976). Determining the number of components from the matrix of partial correlations. Psychometrika, 41, 321-327. https://doi.org/10.1007/BF02293557

Vílchez, J. y Bravo, B. (2015). Percepción del profesorado de ciencia de educación primaria en formación acerca de las etapas y acciones necesarias para realizar una indagación escolar. Enseñanza de las Ciencias, 33(1), 185-202. https://doi.org/10.5565/rev/ensciencias.1529

Wandersee, J., Mintzes, J. J. y Novak, J. D. (1994). Research on Alternative Conceptions in Science. En D. L. Gabel (Ed.), Handbook of Research on Science Teaching and Learning (pp. 177-210). Nueva York: Macmillan Publishing.

Woodhouse, B. y Jackson, P. H. (1977). Lower bounds to the reliability of the total score on a test composed of nonhomogeneous items: II. A search procedure to locate the greatest lower bound. Psychometrika, 42, 579-591. https://doi.org/10.1007/BF02295980

Zimmermann, E. (2000). The Structure and Developing of Science Teachers' Pedagogical Models: Implications for Teacher Eduaction. En J. K. Gilbert y C. J. Boulter (Eds.), Developing Models in Science Education (pp. 325-341). Holanda: Kluwer Academic Publishers. 


\title{
Pre-service teachers' conceptions on the nature of science
}

\author{
José Francisco Serrallé Marzoa, Uxío Pérez Rodríguez, María Asunción Lorenzo Rial, María Mercedes Álvarez Lires \\ Facultad de Ciencias de la Educación y del Deporte. Universidade de Vigo. A Xunqueira, s/n, 36005-Pontevedra \\ jfserralle@uvigo.es, uxio.perez@uvigo.es, marialorenzo@uvigo.es, lires@uvigo.es
}

Many research studies have shown, broadly speaking, that teaching in elementary and secondary education is carried out with a very formal, excessively declarative approach and perspective of scientific content, which presents scientific knowledge as an immutable truth, as a closed result of successive accumulative discoveries and as a constant linear refinement of models and theories.

Teachers' conceptions of the nature of science and technology (NOS) and their views on the relationships between them and the society, as well as on the production of scientific knowledge, interact with their way of teaching and with their ideas about student learning.

To investigate the conceptions about the NOS among teachers in initial training in the degree of Elementary Education, we have designed and validated a questionnaire to evaluate them and, subsequently, to use them as a basis for carrying out didactic proposals.

This paper describes the questionnaire, the sample $(\mathrm{N}=385)$ and the design and validation procedure.

For the construction of the questionnaire, meetings were held with experts who proposed items. In a first selection, a pilot Likert-type questionnaire was formulated with four levels of response, which was administered to a small sample of students of the degree in Elementary Education. Considering the results of the exploratory study, the questionnaire was revised, resulting in a scale of 44 items.

The original sample $(\mathrm{N}=385)$ was divided into two random subsamples. The first was used for an exploratory analysis to construct a model of the main dimensions involved, and the second was used to carry out a confirmatory factor analysis.

The results of the exploratory analysis showed a factor structure with six well-defined dimensions: Positivism, Meta-science, Genesis of Scientific Knowledge, Scientific Methodologies, Research and Validation of Theories. In the analysis, different models were compared, using several fit indices to assess their appropriateness. The best of the models analysed is the one that considers the six factors to be interrelated.

The scale has fair reliability and validity, which leads to the conclusion that it can be useful for research related to this topic.

A cluster analysis of the scores on the scale factors was also performed to segment the cases into groups of similar response profiles and to elaborate «Teacher Types». A division into three types is given, two of which can be categorised as naïve beliefs (Teaching Types 1 and 2) and partially informed beliefs (Teaching Type 3). It is important to highlight the non-appearance of a cluster corresponding to informed beliefs (Schwartz and Lederman, 2008). However, the exploratory and essentially atheoretical nature of the cluster analysis should be emphasised, so that the aim of this study does not go beyond formulating substantiated proposals that may be of interest for future research.

In subsequent studies, we intend to provide detailed descriptive results of the scores on the different factors and to conduct inferential statistical analyses to evaluate the existence of differences of gender, degree, age, and other variables of interest. Another possibility is to use the questionnaire before and after a sustained educational intervention, to assess whether the intervention has contributed to the development of the ideas of the target groups. 
